\title{
A Mortality Case Caused by Thrombotic Microangiopathy after Successful Bloodless Living Donor Liver Transplantation
}

\author{
Sun Young Park', Ho Bum Cho', Gyu Wan You' ${ }^{1}$, Kyeong Sik Kim² \\ Departments of ${ }^{1}$ Anesthesiology and Pain Medicine and ${ }^{2}$ Surgery, Soonchunhyang University Seoul Hospital, Seoul, Korea
}

\begin{abstract}
Thrombotic microangiopathy (TMA) after solid organ transplantation is infrequent and its etiology remains still unclear. Nevertheless, if early diagnosis and early therapies are not performed, it can lead to severe life-threatening complications and even death. A 54-year-old female (a Jehovah's Witness) was diagnosed with drug-induced toxic hepatitis and was decided to undergo bloodless living donor liver transplantation (LDLT). After the successful LDLT, the patient's condition deteriorated, and she was diagnosed with TMA during further evaluation. We tried to proceed with plasma exchange-based treatment, but she and her family declined according to their religious beliefs. The patient expired 4 days after the diagnosis. Physicians should maintain a high level of suspicion for TMA after liver transplantation when clinical manifestations are observed.
\end{abstract}

Keywords: Bloodless medical and surgical procedures; Liver transplantation; Thrombotic microangiopathy; Case report

\section{INTRODUCTION}

Thrombotic microangiopathy (TMA) is defined as clinical features (microangiopathic hemolytic anemia, thrombocytopenia, organ injury) and pathological features (vascular damage and capillary thrombosis) [1]. Risk factors for TMA remain unclear and it is difficult to predict TMA. The incidence rate of TMA after solid organ transplantation was reported as $0.5 \%-3 \%$ and it is most prevalent in kidney transplantation [2]. Though TMA after liver transplantation (LT) is an infrequent but severe life-threatening complication. Plasma exchange was described as a standard treatment for TMA [3].

Jehovah's Witnesses do not permit transfusion of allogeneic blood or blood products including whole blood, red blood cells, platelets, or plasma. So the risks and benefits of any procedure, including any additional risk imposed by blood refusal, should be considered and discussed with the patient. This is significantly important if surgery is a major surgery associated with a large blood volume shift, as LT.

LT requires large surgical dissections and anastomosis of major vessels and most LT patients are at high risk of bleeding because of multiple coagulation disturbances. Therefore, LT in Jehovah's Witness is still challenging and multiple strategies are demanded. Postoperative are also emphasized. Occasionally, in these patients, possible complications during postoperative care can be fatal due to the limitation of treatments.

We present a case of the patient (a Jehovah's Witness) who expired due to TMA after successful bloodless living donor liver transplantation (LDLT). The patient provided written informed consent before the operation for publication about the bloodless LDLT and its prognosis.

\section{CASE REPORT}

A 54-year-old female (weight, $51.2 \mathrm{~kg}$; height, $158 \mathrm{~cm}$ ) of American Society of Anesthesiologists physical status IV was admitted for LDLT. She was a Jehovah's Witness and was diagnosed with drug-induced toxic hepatitis. Four months before the operation, she was already recommended LT, but she and her family refused it because of their religious conviction. Nevertheless, as an acute hepatic failure and hepatic encephalopathy were progressed, she and her family decided to undergo bloodless LDLT. 
Park SY, et al. • Thrombotic Microangiopathy after Liver Transplantation

As a result of hepatic failure, she had stress-induced cardiomyopathy, bilateral pulmonary congestion, hepatorenal syndrome, hepatic encephalopathy. Her model of end-stage liver disease (MELD) score was 37. Preoperatively, the hemoglobin/hematocrit (Hb/Hct) ratio was $11.8 / 34.7$ and the platelet number $48,000 / \mu \mathrm{L}$. We scheduled intraoperative autologous blood transfusion of 500-700 mL via acute normovolemic hemodilution (ANH) and intraoperative blood salvage with cell saver CATS (Continuous Autotransfusion System; Fresenius Kabi AG, Bad Homburg, Germany).

Upon arrival in the operating theater without premedication, standard monitoring devices, including a five-lead electrocardiogram machine; a pulse oximeter; and an oscillometric, noninvasive blood pressure cuff, were applied. General anesthesia was induced using intravenous lidocaine $40 \mathrm{mg}$, propofol $30 \mathrm{mg}$, and rocuronium $0.6 \mathrm{mg} / \mathrm{kg}$ for neuromuscular blockade.

After induction of anesthesia, a 20G arterial cannula (a radial artery catheterization set; Arrow International, Reading, PA, USA) was inserted into the right radial artery to allow continuous arterial blood pressure monitoring and blood sampling. Subsequently, we inserted a multilumen access catheter (a two-lumen central venous access device; Arrow International, Reading, PA, USA) into the right internal jugular vein, followed by inserting a pulmonary arterial catheter (Swan-Ganz; Edwards Lifesciences, Irvine, CA, USA). Additionally, we inserted two 20G catheters (pediatric jugular catheterization sets; Arrow International, Reading, PA, USA) in the right femoral artery and femoral vein for continuous blood pressure monitoring. We monitored intraoperative transesophageal echocardiography due to the cardiomyopathy and various hemodynamic alterations that may occur.

Anesthesia was maintained with oxygen, medical air, and 0.20.6 minimum alveolar concentration of desflurane under monitoring with a bispectral index of 40-60. The total operation time was 6 hours 45 minutes. At the end of the operation, the estimated blood loss during the operation was about 1,200 $\mathrm{mL}$ and her $\mathrm{Hb} /$ Hct ratio was 6.3/18.8, and the platelet number $33,000 / \mu \mathrm{L}$. Two units $(700 \mathrm{~mL})$ of $\mathrm{ANH}$ and $155 \mathrm{~mL}$ of salvaged blood collected from the cell saver were re-infused; in consequence, her $\mathrm{Hb} / \mathrm{Hct}$ ratio was 7.0/21.1 and the platelet number 37,000/ $\mu \mathrm{L}$. After the LDLT had been successfully performed, the patient was transferred to the intensive care unit under sedation without any other events.

On postoperative day (POD) 4, her mental status was nearly alert, and until POD 8, her general condition and vital signs were stable. Postoperative intravenous iron, folate, and vitamin B12 could improve her anemia until POD 10 and her $\mathrm{Hb} / \mathrm{Hct}$ ratio was $8.1 / 25.3$ and the platelet number $44,000 / \mu \mathrm{L}$. However, since then, the anemia and the thrombocytopenia began to deteriorate without bleeding focus. Prophylactic trimethoprim-sulfamethoxazole was stopped because of thrombocytopenia. Ganciclovir due to cytomegalovirus (CMV) infection was also stopped and adjusted again because it could be the cause of anemia and thrombocytopenia. Even with these trials, the patient's condition did not improve.

Further evaluations were done for persistent anemia despite iron replacement therapy. Her laboratory work-up revealed both elevation of lactate dehydrogenase (LDH) 1,891 IU/L (normal $<250 \mathrm{IU} / \mathrm{L}$ ) and reticulocyte count $0.89 \%$ (normal 0.5\%-2.2\%). Peripheral blood smear showed marked spherocytosis with schistocytosis. Coombs test was negative. We could suspect TMA through her laboratory data and peripheral blood morphology at POD 29. At that time, her $\mathrm{Hb} / \mathrm{Hct}$ ratio was 3.1/10.5 and the platelet number $62,000 / \mu \mathrm{L}$. Immediately, we stopped calcineurin inhibitor (CNI) (tacrolimus) and we tried to proceed with plasma exchange-based treatment, but she and her family declined plasma exchange and she expired from cardiac arrest at POD 33.

\section{DISCUSSION}

In this case, we successfully performed bloodless LT according to the patient's religious beliefs, and the immediate recovery process was uneventful. However, the unexpected occurrence of TMA resulted in severe anemia, and the patient refused blood transfusion and plasma exchange, resulting in death.

TMA is clinically characterized by microvascular thrombosis with thrombocytopenia, hemolytic anemia, and red blood cell fragmentation. TMA occurs in a wide range of diseases such as autoimmune diseases, malignant hypertension, vascular rejection, endothelial damage due to drug toxicity, and so on [4]. Possible risk factors of TMA after LDLT were described as anti-human leukocyte antigen antibody positive, disuse of fresh frozen plasma (FFP), splenectomy, female, prolonged anhepatic phase, hepatitis $\mathrm{C}$ virus, and MELD score more than 14 [5]. Additional possible risk factors were immunosuppressive CNI and CMV infection [6]. In this case, she had some potential risk factors like disuse of FFP, female, high MELD score, use of CNI, and CMV infection. How- 
ever, it is difficult to determine what caused TMA because the pathogenesis of TMA is not yet fully understood.

Although the exact etiology of TMA is challenging to determine, it is of considerable clinical importance because of its abrupt onset and high morbidity and mortality without treatment. Treatments of TMA include change of CNI, plasma exchange, supportive packed red blood cells transfusion, and experimental monoclonal antibodies therapies [7]. Without effective treatment, the mortality rate of TMA was up to $90 \%$. However, the introduction of plasma exchange over FFP infusion has improved the survival rate dramatically to $80 \%-90 \%$ [8]. Another study presented the response rate of TMA to treatment by plasma exchange with and CNI change was estimated to be as high as $87 \%$ [5].

CNI change means discontinuation, dose reduction or conversion with concurrent FFP infusion, or plasma exchange. In mild forms of TMA, discontinuation of the CNI may suffice [9]. But in our case, discontinuation of CNI only could not improve her state. Recently, a humanized monoclonal antibody (eculizumab) that is reported as effective to treat TMA was considered, but the treatment is effective when combined with plasma exchange [10]. Furthermore, without confirmation of ADAMTS13 (a disintegrinlike and metalloprotease with thrombospondin type 1 motif, member 13) levels, the use of eculizumab had significant costing, so we should wait for approval of the Korean Health Insurance and Review Assessment Service. Meanwhile, the patient's condition deteriorated, and she expired 4 days after diagnosis.

We presented a mortality case caused by TMA after successful bloodless LDLT. Physicians should maintain a high level of suspicion for TMA when the unexplained elevation of $\mathrm{LDH}$ and progressive thrombocytopenia in LT recipients. It is challenging to predict TMA, but early diagnosis and early initiation of intensive therapies are crucial to improve the prognosis. Especially in the case of Jehovah's Witness, further studies should be performed about alternative treatment options for TMA.

\section{CONFLICT OF INTEREST}

No potential conflict of interest relevant to this article was reported.

\section{REFERENCES}

1. George JN, Nester CM. Syndromes of thrombotic microangiopathy. N Engl J Med 2014;371:654-66.

2. Miyata R, Shimazu M, Tanabe M, Kawachi S, Hoshino K, Wakabayashi G, et al. Clinical characteristics of thrombotic microangiopathy following ABO incompatible living donor liver transplantation. Liver Transpl 2007; 13:1455-62.

3. Rock GA, Shumak KH, Buskard NA, Blanchette VS, Kelton JG, Nair RC, et al. Comparison of plasma exchange with plasma infusion in the treatment of thrombotic thrombocytopenic purpura. Canadian Apheresis Study Group. N Engl J Med 1991;325:393-7.

4. Benz K, Amann K. Pathological aspects of membranoproliferative glomerulonephritis (MPGN) and haemolytic uraemic syndrome (HUS)/ thrombocytic thrombopenic purpura (TTP). Thromb Haemost 2009; 101:265-70.

5. Shindoh J, Sugawara Y, Akamatsu N, Kaneko J, Tamura S, Yamashiki N, et al. Thrombotic microangiopathy after living-donor liver transplantation. Am J Transplant 2012;12:728-36.

6. Tamura S, Sugawara Y, Matsui Y, Kishi Y, Akamatsu N, Kaneko J, et al. Thrombotic microangiopathy in living-donor liver transplantation. Transplantation 2005;80:169-75.

7. Verbiest A, Pirenne J, Dierickx D. De novo thrombotic microangiopathy after non-renal solid organ transplantation. Blood Rev 2014;28:269-79.

8. Bell WR, Braine HG, Ness PM, Kickler TS. Improved survival in thrombotic thrombocytopenic purpura-hemolytic uremic syndrome: clinical experience in 108 patients. N Engl J Med 1991;325:398-403.

9. Paramesh AS, Grosskreutz C, Florman SS, Gondolesi GE, Sharma S, Kaufman SS, et al. Thrombotic microangiopathy associated with combined sirolimus and tacrolimus immunosuppression after intestinal transplantation. Transplantation 2004;77:129-31.

10. Legendre CM, Licht C, Muus P, Greenbaum LA, Babu S, Bedrosian C, et al. Terminal complement inhibitor eculizumab in atypical hemolytic-uremic syndrome. N Engl J Med 2013;368:2169-81. 